\title{
RECUPERAÇÃO DE ÁREAS CONTAMINADAS POR PESTICIDAS ORGANOCLORADOS NA CIDADE DOS MENINOS, MUNICÍPIO DE DUQUE DE CAXIAS, RJ ${ }^{1}$
}

\author{
Daniela Buosi², Jeanine Maria Felfili ${ }^{3}$
}

\begin{abstract}
RESUMO - O Serviço Nacional de Malária operacionalizou uma fábrica de pesticidas organoclorados utilizados em campanhas de saúde pública (HCH e DDT) entre 1950 e 1965, na Cidade dos Meninos, no município de Duque de Caxias, RJ, visando combater doenças rurais endêmicas. Após denúncia na mídia, em 1989, a FEEMA detectou que, com a desativação da fábrica, foram deixados resíduos da produção dos pesticidas, causando a contaminação do solo. Neste trabalho foram realizadas análises foliares e de solo em dois talhões de espécies de eucalipto (Eucalyptus grandis e Corymbia citriodora (Hook.) K.D. Hill \& L.A.S. Jhonson), com o objetivo de avaliar o potencial da espécie como fitorremediador de área contaminada por organoclorados. Coletaramse cinco amostras compostas de folhas (três do C. citriodora e duas do E. grandis) e uma composta de solo em cada um dos talhões estudados para análise de organoclorados por cromatografia gasosa. As alturas médias dos talhões de E. grandis e C. citriodora foram, respectivamente, de 9,1 e 16,8 com diâmetros médios de 7,8 e 15,95 cm; a média de C. citriodora foi similar à de outros plantios em regiões de mata atlântica, ficando a de E. grandis pouco abaixo. Detectou-se a presença de organoclorados nas folhas analisadas, porém em baixas concentrações. Nos solos, as concentrações encontradas foram altas, sendo maiores no talhão de $E$. grandis. Os resultados foram comparados com análises prévias na área, indicando que as espécies atuaram como fitorremediadoras, especialmente o C. citriodora, uma vez que houve redução na contaminação do solo e absorção dos organoclorados pelas folhas.
\end{abstract}

Palavras-chave: Pesticidas, fitorremediação, eucalipto.

\section{RECOVERY OF SOILS CONTAMINATED BY ORGANOCHLORINE PESTICIDES IN CIDADE DOS MENINOS, DUQUE DE CAXIAS, RJ, BRAZIL}

\begin{abstract}
The Malaria National Service built a factory of organoclorine pesticides used in public health campaigns (HCH and DDT) between 1950 and 1965, at Cidade dos Meninos, in the municipal district of Duque de Caxias/RJ to control the dissemination of endemic rural diseases. After a media outcry, in 1989, FEEMA investigated and found out that after the factory closure piles of residues were left behind causing the contamination of the soils. Analyses of foliar tissues and soil were conducted in two stands of Eucalyptus (Eucalyptus grandis e Corymbia citriodora (Hook.) K.D. Hill \& L.A.S. Jhonson), with the objective of evaluating the potential of the species as phytoremediator of contaminated soils by organochlorines pesticides. A composite soil sample and five samples of leaves were collected in each one of the studied places. Three leaf samples from C. citriodora and two from E. grandis were analized for pesticides organochlorines by gas chromatography. Both species grew on the same conditions, with average heights of 9,1 e 16,8 $\mathrm{m}$ and average diameters of 7,8 and 15,95 cm for E. grandis and C. citriodora, respectively. The average height and diameter of C. citriodora were similar to those found for other stands in the Atlantic forest region while the E. grandis was a little bellow those averages. The presence of organochlorine pesticides was detected in the leaves at low concentrations but the concentrations in the soils were high, being greater in the E. grandis plantation. These results were compared to previous analyses and suggested that the species can function as phytoremediators especially the C. citriodora.
\end{abstract}

Key words: Pesticides, phytoremediation, eucalypt, Brazil

\footnotetext{
${ }^{1}$ Recebido para publicação em 03.7.2002 e aceito para publicação em 08.6.2004.

${ }^{2}$ Mestre em Ciências Florestais, Departamento de Engenharia Florestal da Universidade de Brasília, Cx.Postal 045357, 70919-970 Brasília,DF.

${ }^{3}$ Professora do Departamento de Engenharia Florestal da UnB.
} 


\section{INTRODUÇÃO}

A descoberta das propriedades inseticidas do DDT (1940) e do HCH (1940-1942) deu um grande impulso no combate aos insetos, de modo que, a partir da década de 1940, os inseticidas organoclorados foram amplamente utilizados na agricultura, na indústria pecuária e nos programas de combate a insetos transmissores de doenças e nas campanhas de saúde pública (HAYES, 1975).

Em 1949, o diretor do extinto Serviço Nacional de Malária, vinculado ao então Ministério da Educação e Saúde, solicitou à Fundação Abrigo Cristo Redentor, educandário que albergava menores carentes, o empréstimo de oito pavilhões desocupados, nessa época, para a instalação temporária do Instituto Nacional de Malariologia (OLIVEIRA, 1994).

Visando à auto-suficiência na produção de pesticidas utilizados em campanhas de saúde pública e considerando a avassaladora disseminação da malária no país, aliada à oportunidade de acesso à tecnologia proporcionada pela chegada ao Brasil de um químico holandês, Henk Kemp, conhecedor do processo industrial de produção de $\mathrm{HCH}$, o Instituto de Malariologia promoveu a construção de uma unidade fabril na Cidade dos Meninos (OLIVEIRA, 1994).

Não há como negar os benefícios que esses produtos de ampla ação e persistência trouxeram ao homem (PASCHOAL, 1979). Porém, em 1962, Carson publicou o livro "Primavera Silenciosa", relatando que o amplo uso dos organoclorados poderia ser a causa da diminuição da população de diversas aves.

Uma das características indesejáveis desses compostos, do ponto de vista ambiental, é a persistência, que consiste na capacidade das substâncias em permanecer inalteradas e ativas por muito tempo no solo, na água e nos alimentos (BASTOS, 1999).

Por razões econômicas, a fábrica de produção de HCH em Cidade dos Meninos foi desativada entre os anos de 1960 e 1965, permanecendo no local todo o seu acervo abandonado, inclusive os estoques e resíduos da produção fabril (OLIVEIRA, 1994).

Em 1989, segundo denúncias dos meios de comunicação, o poder público foi acionado para investigar possível contaminação ambiental proveniente da Cidade dos Meninos.

R. Árvore, Viçosa-MG, v.28, n.3, p. 465-470, 2004
Após a condução de vários estudos ambientais inconclusivos na área, o Ministério da Saúde decidiu proceder, por intermédio da empresa Nortox S.A., à neutralização do $\mathrm{HCH}$, em uma tentativa de remediar a contaminação do solo, por meio da aplicação de cal virgem (CaO) (OLIVEIRA et al., 1997). Por essa ação, a composição inicial dos resíduos foi modificada, gerando novos compostos e novos riscos. A empresa que executou a intervenção apresentou relatório mostrando a diminuição proporcional de $\gamma-\mathrm{HCH}$, mas não quantificou o isômero nem apresentou qualquer avaliação do restante dos compostos existentes (BASTOS, 1999).

Associado à aplicação da cal e à agregação de adubo orgânico ao solo, a empresa Nortox S.A. sugeriu o plantio de espécies florestais na área contaminada, visando a uma biorremediação associada ao tratamento químico, com vistas a promover a ciclagem de nutrientes (LASAT, 2002). A espécie eucalipto foi, então, escolhida em razão do seu rápido crescimento.

Neste trabalho, partiu-se da premissa de que o plantio florestal com eucalipto funcionaria como biorremediador em área contaminada por organoclorado e teve como objetivos: analisar a contaminação do solo na área com plantio de eucalipto; comparar a contaminação do solo atual na área plantada com a dessa área antes do plantio; e analisar algumas características do plantio em comparação com outros plantios em áreas saudáveis.

\section{MATERIAL E MÉTODOS}

A área objeto deste estudo está inserida na localidade da Cidade dos Meninos, no município de Duque de Caxias, RJ, e é denominada "área foco principal de contaminação".

O plantio, com aproximadamente $12.000 \mathrm{~m}^{2}$, onde as amostras foram coletadas, era formado exclusivamente de eucaliptos, divididos em dois talhões, um de Eucalyptus grandis (área 1, com $5.000 \mathrm{~m}^{2}$ ) e outro de Corymbia citriodora (área 2, com $6.900 \mathrm{~m}^{2}$ ). Não foi obedecido um espaçamento regular no plantio das mudas, porém se constatou que o espaçamento mais freqüente foi o de $1,0 \times 1,5 \mathrm{~m}$.

Foram coletadas duas amostras de solo, sendo uma representativa de cada área onde se localizavam os talhões de eucalipto. A coleta foi realizada com a 
utilização de duas sondas distintas: a sonda "Wacker Hammer", usada para abrir um furo de duas polegadas de diâmetro, onde foi introduzida a segunda sonda ("Bosch"), que possuía um amostrador de $50 \mathrm{~cm}$ de comprimento, também conhecido como "liner". À medida que o amostrador vai sendo cravado no solo, ocorre o seu preenchimento gradual pelo material a ser amostrado. Para evitar contaminação cruzada, cada amostra é retirada dentro de um "liner” individual.

Em 2001, quando o plantio tinha sete anos, foram coletados dados de altura, com a utilização de um hipsômetro de Haga, e de diâmetros (DAP - diâmetro à altura do peito), com o auxílio de uma suta, de todos os indivíduos do talhão, bem como anotadas as ocorrências de mortalidade no plantio.

Segundo Bataglia e Dechen (1986), as folhas são consideradas o foco das atividades fisiológicas dentro das plantas. Dessa forma, a matriz folha foi selecionada para verificação de acúmulo de compostos organoclorados pelos eucaliptos. Após a coleta, em setembro de 2001, as folhas foram lavadas com água destilada e secadas em estufa.

Os indivíduos foram agrupados por classe de altura, sendo selecionados aqueles da classe mais representativa e da classe mais alta. Da espécie E. grandis foram selecionados indivíduos da classe com 6,1 a 8,0 m (denominados Folha 4) e acima de 12 m (Folha 5) já da espécie C. citriodora foram selecionados indivíduos da classe com 16,1 a 20 m (Folha 1), acima de $20 \mathrm{~m}$ (Folha 2), correspondendo ao critério adotado para a amostragem. Por ser esta espécie mais representativa em número de indivíduos, foram coletadas amostras na classe com 12,1 a 16 m (Folha 3).

Em cada uma das classes selecionadas foram coletadas as folhas de cinco indivíduos, para a formação de amostras compostas. Colheram-se folhas de todas as posições da copa, apenas folhas sadias. Para comparar os valores obtidos com os de indivíduos plantados em áreas não-contaminadas (backgound), foram coletadas "amostras-controle" (denominadas Folha 6 e 7), sendo uma amostra para cada uma das espécies de eucalipto em questão. As amostras foram coletadas em uma fazenda localizada no núcleo rural Boqueirão em Brasília, DF. O plantio da área-controle estava com oito anos e com espaçamento de 1,5 x 1,5 m, à época da coleta, em setembro de 2001.

As análises de determinação dos resíduos de organoclorados em solo e folhas foram feitas por cromatografia gasosa de alta resolução acoplada à espectrometria de massas, no Laboratório Analytical Solution, no Rio de Janeiro.

As análises físicas e químicas para caracterização dos solos foram realizadas no laboratório da Empresa Brasileira de Pesquisas Agrícolas (EMBRAPA), no Rio de Janeiro.

As comparações de teores de compostos químicos no solo foram feitas com os resultados obtidos antes e depois do tratamento químico do solo, através da aplicação de cal (OLIVEIRA, 1994; BASTOS, 1999).

\section{RESULTADOS E DISCUSSÃO}

O plantio de eucalipto consistiu de um talhão de 1.074 indivíduos da espécie Corymbia citriodora e outro de 693 indivíduos de Eucalyptus grandis, com aproximadamente sete anos de idade cada talhão.

Os indivíduos de E. grandis apresentaram-se distribuídos em cinco classes de altura, sendo a mais representativa em número de indivíduos a de 6,1 a 8,0 m. A média de altura da espécie foi de 9,1 m. A espécie C. citriodora foi distribuída em quatro classes de altura, sendo a com o maior número de indivíduos a que compreendia os de 16,1 a $20 \mathrm{~m}$ de altura. A média de altura da espécie foi de 16,8 m.

As comparações quanto ao desenvolvimento das espécies foram efetuadas com dados de literatura para plantios dessas espécies. Comparando o plantio de E. grandis e C. citriodora da Cidade dos Meninos com os realizados em outras localidades, as médias de altura encontradas em C. citriodora mostraramse similares aos valores dos demais plantios. Já a espécie $E$. grandis obteve média abaixo do valor encontrado em outras regiões (EMBRAPA, 1980; CHAVES, 1997; SIMÕES e FERREIRA, 1997; STAPE e BENEDETTI, 1997).

A distribuição diamétrica da espécie $E$. grandis resultou em cinco classes de diâmetro, sendo as mais representativas as de 4,1 a $6,0 \mathrm{~cm}$ e 8,1 a $10,0 \mathrm{~cm}$. A espécie C. citriodora (Figura 12) apresentou cinco classes de diâmetro, sendo a de 14,1 a 18,0 em a mais representativa.

Da mesma forma como discutido com relação à altura dos indivíduos, a espécie $E$. grandis obteve

R. Árvore, Viçosa-MG, v.28, n.3, p. 465-470, 2004 
média abaixo daquelas encontradas em outras regiões (EMBRAPA, 1980; CHAVES, 1997; SIMÕES e FERREIRA, 1997; STAPE e BENEDETTI, 1997). Aespécie C. citriodora apresentou média diamétrica de 15,95 $\mathrm{cm}$, sendo similar à de outros plantios.

No estudo de mortalidade conduzido, a espécie C. citriodora apresentou $27,3 \%$ de mortalidade e a espécie E. grandis, 53,4\%. A taxa de mortalidade apresentada pela espécie C. citriodora é comparável à mensurada em outras localidades (27,7 e 29\%). Já a espécie $E$. grandis apresentou mortalidade superior à das demais encontradas em outras localidades. Porém, deve-se ressaltar que a mortalidade é um fator que depende muito de tratos culturais.

As comparações de alturas e diâmetros com outros plantios fornecem indicações de que a contaminação não inibiu o crescimento das espécies, que apresentaram um desenvolvimento compatível com aquele encontrado em outras áreas, em especial no que se refere ao C. citriodora. Mesmo considerando as diferenças de condições ambientais e tratos culturais, pôde-se verificar que as espécies se desenvolveram na área contaminada dentro dos limites encontrados em outros plantios.

As análises físico-químicas das amostras de solo da área de estudo indicaram que o solo apresenta $\mathrm{pH}$ igual a 8,2 e 8,4, respectivamente, nas amostras de solo 1 (talhão de E. grandis) e 2 (talhão de $C$. citriodo$r a$ ). Tais resultados indicam que o solo sob o tratamento partiu de uma condição original de $\mathrm{pH}$ entre 4,6 4,8 (OLIVEIRA, 1994) para um nível de 8,0 (BASTOS, 1999), por ocasião da aplicação de cal e adubo orgânico. Atualmente, sete anos após o plantio de eucalipto, apesar da agregação de matéria orgânica e do desenvolvimento do plantio, o solo permanece básico, condição desfavorável à retenção dos compostos organoclorados.

O teor de matéria orgânica nas amostras de solo (5,29 e 7,34\%) foi elevado. Estudos anteriores (OLIVEIRA, 1994; BASTOS, 1999) haviam apontado valores de 2,17 e 1,39\%. Essa condição favorece a retenção de compostos organoclorados no solo, sendo um resultado positivo da biorremediação, sendo a contaminação maior na área com plantio de E. grandis. A área com plantio de C. citriodora possui maior conteúdo de matéria orgânica e solo menos argiloso, o que auxilia a decomposição de compostos organoclorados.
Em relação à granulometria, as amostras analisadas apresentaram maior teor de areia grossa (grãos entre 2 e $0,2 \mathrm{~mm}$ ). O solo 2 (talhão de C. citriodora) apresentou maior teor de argila $(<0,002 \mathrm{~mm})$ e silte $(0,05$ e $0,002 \mathrm{~mm}$ ).

Na amostra de solo da área 1 (talhão de E. grandis) não foram detectados os compostos hexaclorobenzeno e pentaclorofenol. Os demais compostos detectados pela análise apresentaram-se acima dos padrões estabelecidos pela legislação holandesa $(2.000 \mu \mathrm{g} / \mathrm{kg})$, sendo a maior concentração encontrada de $\alpha-\mathrm{HCH}$ igual a $89.467,56 \mu \mathrm{g} / \mathrm{kg}$, seguida de $\beta-\mathrm{HCH}, \gamma-\mathrm{HCH}$ e $\delta$ - $\mathrm{HCH}$ (23.645,14 $\mu \mathrm{g} / \mathrm{kg}, 32.244,09 \mu \mathrm{g} / \mathrm{kg}$ e $7.185,73 \mu \mathrm{g} / \mathrm{kg}$, respectivamente). Todos os isômeros de HCH apresentaram teores elevados de concentração. No resultado das análises da concentração de DDT $_{\text {Total, }}$ expressa pela soma dos seus isômeros e dos seus metabólitos (DDD e DDE), observou-se concentração inferior à máxima permitida pelas normas internacionais (2.000 $\mu \mathrm{g} / \mathrm{kg}$ ), ou seja, $467,75 \mu \mathrm{g} / \mathrm{kg}$. Os demais compostos analisados, apesar de terem sido detectados nas amostras, exibiram baixas concentrações. Na área 2 (talhão de C. citriodora) também foram encontrados todos os compostos, excetuando-se hexaclorobenzeno e pentaclorofenol, porém em concentrações muito mais baixas do que na área 1 , sendo a maior concentração encontrada de $\beta-\mathrm{HCH}$ igual a $5.792,60 \mu \mathrm{g} / \mathrm{kg}$, que foi aproximadamente mais de duas vezes superior ao permitido por normas internacionais. O resultado das análises da concentração de $\mathrm{DDT}_{\text {Total }}$ (soma dos isômeros e metabólitos) apresentou concentração igual a 336,80 $\mu \mathrm{g} / \mathrm{kg}$, inferior ao limite permitido pelos padrões internacionais $(2.000 \mu \mathrm{g} / \mathrm{kg})$.

Detectou-se uma redução na concentração dos isômeros de $\mathrm{HCH}$ da ordem de centenas a milhares de vezes (2,9 x $10^{3} \mu \mathrm{g} / \mathrm{kg}$ a $\left.6,2 \times 10^{6} \mu \mathrm{g} / \mathrm{kg}\right)$, quando comparados os resultados deste com os de Bastos (1999), e da ordem de dezenas a centenas de vezes (3.170 $\mu \mathrm{g} / \mathrm{kg}$ a $191.610 \mu \mathrm{g} / \mathrm{kg}$ ), em comparação com os de Oliveira (1994).

Os resultados das amostras de solo e de folha estão apresentados no Quadro 1.

Nas análises de folhas de E. grandis e C. citriodora, coletadas na fazenda do núcleo rural Boqueirão, em Brasília, DF, e utilizadas como controle, não foi detectada nenhuma concentração dos compostos em questão, indicando, portanto, que a presença de 
Quadro 1 - Resultados das análises de pesticidas em amostras compostas de folhas e solo na Cidade dos Meninos, município de Duque de Caxias, no Rio de Janeiro, 2001

Table 01 - Results of the pesticide analyses in composite samples of leaves and soils in Cidade dos Meninos, Duque de Caxias municipality in Rio de Janeiro, Brazil

\begin{tabular}{lccccccccc}
\hline COMPOST0 & Folha 1 & Folha 2 & Folha 3 & Folha 4 & Folha 5 & Folha 6 & Folha 7 & Solo 1 & Solo 2 \\
\hline 1,2,4-Triclorobenzeno & 12,94 & 34,33 & 34,83 & 0,63 & 102,70 & ND & ND & 179,23 & 22,77 \\
2,4,6-Triclorofenol & ND & ND & ND & ND & ND & ND & ND & 220,18 & 19,92 \\
2,4,5-Triclorofenol & ND & ND & ND & ND & ND & ND & ND & 8,83 & 3,30 \\
Alfa-Hexaclorocicloexano & ND & ND & ND & 1164,70 & ND & ND & ND & 89467,56 & 3206,08 \\
Hexaclorobenzeno & ND & ND & ND & ND & ND & ND & ND & ND & ND \\
Beta-Hexaclorocicloexano & ND & ND & ND & ND & ND & ND & ND & 23645,14 & 5792,60 \\
Gama-Hexaclorocicloexano & ND & ND & ND & ND & ND & ND & ND & 32244,09 & 503,39 \\
Delta-Hexaclorocicloexano & ND & ND & ND & ND & ND & ND & ND & 7185,73 & 454,57 \\
Pentaclorofenol & 7,53 & 7,53 & 7,53 & 7,53 & ND & ND & ND & ND & ND \\
o,p-DDE & ND & ND & ND & ND & ND & ND & ND & 2,42 & 4,65 \\
p,p-DDE & 12,25 & 12,21 & ND & ND & 41,03 & ND & ND & 99,36 & 87,34 \\
o,p-DDD & ND & 42,89 & ND & ND & ND & ND & ND & 8,96 & 24,25 \\
p,p-DDD & 22,23 & 24,78 & ND & ND & ND & ND & ND & 47,88 & 68,26 \\
o,p-DDT & ND & ND & 91,27 & 23,56 & ND & ND & ND & 47,34 & 29,90 \\
p,p-DDT & ND & ND & ND & ND & ND & ND & ND & 261,79 & 122,40 \\
\hline
\end{tabular}

ND - não detectável.

Concentração em ppb (ì g/kg amostra).

Limite de detecção para todos os compostos: 0,01 ì g/kg

Compostos o,p-DDE, o,p-DDD e o,p-DDT foram quantificados pelo método semiquantitativo.

Folha 1 - amostra de folhas de Corymbia citriodora - indivíduos de 16,1 a 20,0 m de altura.

Folha 2 - amostra de folhas de Corymbia citriodora - indivíduos acima de 20,0 m de altura.

Folha 3 - amostra de folhas de Corymbia citriodora - indivíduos de 12,1 a 16,0 m de altura.

Folha 4 - amostra de folhas de Eucalyptus grandis - indivíduos de 6,1 a 8,0 m de altura.

Folha 5 - amostra de folhas de Eucalyptus grandis - indivíduos acima de $12 \mathrm{~m}$ de altura.

Folha 6 - amostra de folhas de Eucalyptus grandis - controle.

Folha 7 - amostra de folhas de Corymbia citriodora - controle.

Solo 1 - amostra de solo coletada na área de plantio do Eucalyptus grandis.

Solo 2 - amostra de solo coletada na área de plantio do Eucalyptus citriodora.

organoclorados nas folhas dos eucaliptos pode ser atribuída a absorção desses compostos presentes no solo contaminado.

Nas folhas de E. grandis, coletadas na área foco principal de contaminação na Cidade dos Meninos, foram detectados os compostos DDE e triclorobenzeno (amostra 5) e $\alpha-\mathrm{HCH}$, pentaclorofenol e DDT (amostra 4). As concentrações foram da ordem de dezenas de $\mathrm{mg} / \mathrm{kg}$, com exceção da concentração de $\alpha-\mathrm{HCH}$ na amostra $4(1.164,70 \mu \mathrm{g} / \mathrm{kg})$.

Nas folhas de C. citriodora foram detectados, em todas as amostras, os compostos triclorobenzeno e pentaclorofenol; nas amostras 1 e 2 DDE e DDD, respectivamente, além de DDD na amostra 2 e DDT na amostra 3. As concentrações foram similares às encontradas na espécie $E$. grandis.

\section{CONCLUSÕES}

A contaminação do solo na área anos após a desativação da fábrica ainda é alta, apesar de apresentar teores mais baixos do que os encontrados em trabalhos anteriores, o que evidencia a função da espécie como fitorremediador.

As análises foliares indicaram a absorção de compostos de pesticidas pelas espécies $E$. grandis e C. citriodora, porém em baixas concentrações. 
O desenvolvimento de espécies de eucalipto nos solos contaminados por organoclorados aponta o potencial dessas espécies para atuarem como fitorremediadores, tanto na absorção de compostos organoclorados quanto na melhoria das condições do solo pela ciclagem de nutrientes e pelo aporte de matéria orgânica.

\section{REFERÊNCIAS BIBLIOGRÁFICAS}

\section{BASTOS, L.H. Investigação da \\ contaminação do solo por \\ organoclorados, na Cidade dos Meninos, Duque de Caxias, RJ, avaliação dentro de um novo cenário, após adição de cal. 1999. 175 f. Dissertação (Mestrado em Engenharia Florestal) - Escola Nacional de Saúde Pública da Fundação Oswaldo Cruz, Rio de Janeiro, 1999.}

BATAGLIA, O.C.; DECHEN, A.R. Critérios alternativos para diagnose foliar. In: SIMPÓSIO AVANÇADO DE QUÍMICA E FERTILIDADE DO SOLO, 1986, Piracicaba. Resumos... Piracicaba: [s.n.], 1986. p.115-136.

CARSON, R. Silent spring. New York: Houghton Mifflin Company, 1962. 368 p.

CHAVES, R. Espaçamento em Eucalyptus. In: CONFERENCE ON SILVICULTURE AND IMPROVEMENT OF EUCALYPTS, 1997.

Resumos... [S.l.: s.n.], 1997. p. 180 - 183.

EMBRAPA. Avaliação de espécies e procedências de Eucalyptus em Minas Gerais e Espirito Santo. [S.l.: s.n.], 1980. 69 p. (Boletim de Pesquisa, 1.)
HAYES, W.A. Toxicology of pesticides. Baltimore: The Williams and Wilkins Company, 1975. $156 \mathrm{p}$.

LASAT, M.M. Phytoextraction of toxic metals - A review of biological mechanisms. Journal of Environmental Quality, v. 31, p. 109-120, 2002.

OLIVEIRA, R.M. Estudo da contaminação do solo e pasto por hexaclorociclohexano (HCH) na Cidade dos Meninos em Duque de Caxias, RJ. 1994. 125 f. Dissertação (Mestrado em Engenharia Florestal) - Escola Nacional de Saúde Pública da Fundação Oswaldo Cruz, Rio de Janeiro, 1994.

OLIVEIRA, C.C.T.; RODRIGUES, J.J.; NASCIMENTO, M.F.M. Cidade dos meninos: estudo de caso. Rio de Janeiro: Universidade Federal do Rio de Janeiro, 1997. 102 p.

PASCHOAL, A.D. Pragas, praguicidas e a crise ambiental: problemas e soluções. Rio de Janeiro: [s.n.], 1997. 102 p.

SIMÕES, J.W.; FERREIRA, M. Resultado do desbaste em 14 espécies de eucalipto para produção de madeira e sementes. In: CONFERENCE ON SILVICULTURE AND IMPROVEMENT OF EUCALYPTS, 1997. Resumos ... [S.1.: s.n.], 1997. p.343-348.

STAPE, J.L.; BENEDETTI, V. Decréscimo de produtividade e respostas da brotação do Eucalyptus grandis à fertilização com macronutrientes em areia quartzosa no Estado de São Paulo - Brasil. In: CONFERENCE ON SILVICULTURE AND IMPROVEMENT OF EUCALYPTS, 1997. Resumos ... [S.l.: s.n.], 1997. p. 112-117. 